\title{
Server Based Wireless Motion Detection System Using PIR Sensor
}

\author{
Ms. Shruti Timande \\ Department of Computer Engineering BDCOE Sewagram India \\ shruti.wcc.tgp@gmail.com
}

\begin{tabular}{|l}
\hline Article History \\
Article Submission \\
21 May 2013 \\
Revised Submission \\
17 July 2013 \\
Article Accepted \\
15 August 2013 \\
Article Published \\
30 September 2013
\end{tabular}

Abstract
Detecting human movement plays a vital in this industrial era where it can be
effectively used in industrial sectors dealing with reactors and boilers. A server based
automated system that allows only the authorized persons to enter into the hazardous
zone that will avoid misguiding of the system by unknown persons. In this research
article, a server based motion detection module involving PIR sensor and GSM is
proposed. Here we use two modules one is digital image processing and another one
is embedded system. It is used to detect any obstacle in within its field. Once obstacle
will be detected camera will be on. Camera captured an upcoming image and check
whether it is a human or animal, if it is a human it will compare with input database
images, then easily find unauthorized person.

Keywords: PIR, Sensors, Server, GSM, Industrial sector

\section{Introduction}

The server based automated type of system provides more security and can provide applicability at banks, offices and secure places where the access to a system should be maintained more secure. The system can also be used for many other critical applications requiring tamper free Identification of the person [3] [4]. The system here we are using a PIC 16F877A Microcontroller for which s and GSM modem are connected. In this project s are used to detect the motion of human beings entering into the high security zones. After detecting, this system will intimate the authorized people by sending an SMS using GSM (Global system for mobile communication) modem. We are using both Digital image processing and embedded system modules to complete this proposed system.

In Digital image processing we take collection of database images as input. Then we normalized all input images for getting unique input. Now we extract some of the features of the image using princomp function. After extraction take one test image and test that image with collection of database images. If the test image and database image is not matched the unauthorized person is easily identified [5]. Using this testing procedure we identify the unauthorized person. GSM (Global System for Mobile Communication) is used to send the message after identification of unauthorized person. Princomp function is used to extract some important features of the face. It takes the triangular shape of the face to extract the features that is each and every human being has eyes and nose [6].

Triangular shape means it takes eyes and nose to extract features. Human beings eyes and nose shape does not change even their getup will be changed like hairless, more beard etc. Camera is used in this project for some extra features. This coding will be very helpful to compare system database images with newly entered person. Then unauthorized person will be identified and message sends to the authorized person through GSM modem [7].

\section{Related Work}

In existing system, the entry of the authorized person is authenticated with a RF transmitter at the person by which all the details of the person are obtained through receiver at the spot. But in proposed system, it does not have any 
transmitter and receiver. It has a small Fresnel lens inside the sensor. That small chip used to detect the obstacle. Also does not emit any energy while sensing. Another problem in this system is here GSM is not used. But in our proposed system we use GSM. After finding the unauthorized person with the help of GSM we sent the message through GSM modem. Also we interface camera with the system database, so we easily identified the unauthorized person through the camera .In existing system, sense motion in any kind.so it senses human, animal, vehicle, and any object. So after detection we don't know whether it is a human or object.so we overcome this problem using camera. We develop some mat lab coding to identify whether the entered person is a human or animal.

A new efficient approach for "Target detection and classification using PIR Sensor and Seismic Sensor", proposed by Sarkaretal [1] in the year 2012 brings the concept of detecting the particular target by using seismic and PIR Sensor. A set of ground sensors are employed to detect intruders in red zone where false alarm reate is very high due to limitations in computation techniques [8]. Researchers toiled to identify motion of human through wavelet based target detection method with fast execution time and low memory requirements. Simulated results shows PIR sensors are better than seismic sensors for movement oriented identification. A new efficient approach using pyro-electric sensor for motion detection proposed by Piero_Zappietal [2], in the year 2010 brings the concept of distance detection of unauthorized person movement using PIR Sensor. However this work failed to detect multiple human detection. Future works concentrated on feature extraction, sensor fusion and employment of wireless sensor nodes with reduced computational time and complexity [9][10]. The efficiency of the above existing methods ranges between $60 \%$ to $74 \%$.

\section{Proposed System}

In digital image processing we use some collection of database images as input. In that database all the input images having same sizes. Sometimes sizes are not necessary, because if we take white and white image, it has some pixel size. Similarly if we take color images it has some other pixel sizes. The hardware part of the system consists of controller, GSM modem, and camera. The unauthorized person is easily identified using Mat lab code in digital image processing. In embedded system the Microcontroller is connected with PIR Sensors, RS 232 converter, LCD display, and GSM modem. The System block diagram is shown in the figure 1.

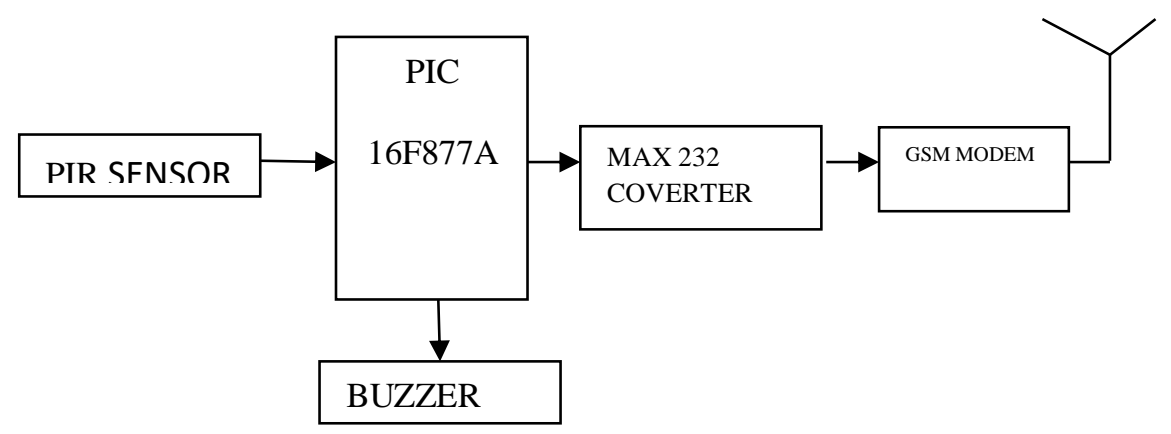

Fig.1: Block Diagram of the proposed system

The detected signal is given to the PIC Microcontroller. Then it gives that detected signal to the MAX 232 level converter. Max 23 level converters is used to convert any output voltage to TTL(Transistor-Transistor Logic).Because the output signal voltage level is high means power supply design will be complicated. So to avoid that problem we use MAX 232 level converters. Step down transformers also used to convert 230 volts AC to 15 volt output signal. Now if any obstacle is detected by PIR Sensor it will be displayed on LCD display as "PIR Sensor obstacle detected". Then Microcontroller sent the message through GSM modem. Now we received a message from GSM modem as some obstacle is detected. Suppose, if an entered person is human, Mat lab coding is enabled and it will check entered 
person image with camera database images. If both the images are not matched the unauthorized person is identified. Identification message will sent by Microcontroller through GSM modem and also if the unauthorized person is identified alarm also ON. The PIR sensor operation is tabulated in table 1.

\section{Table 1: Pin Description of PIR Sensor}

\begin{tabular}{|l|l|l|}
\hline Pin & Name & Functions \\
\hline $\mathbf{+ 5 V}$ & VCC & Connect to +5V DC regulated power supply \\
\hline OUT & Output & $\begin{array}{l}\text { Output is HIGH when idle (no motion detected). Output remains LOW } \\
\text { when Sensor is triggered. LCD Display is used to know the current } \\
\text { status. }\end{array}$ \\
\hline GND & Ground & Power Supply Ground: 0V \\
\hline
\end{tabular}

\section{Simulation Results \& Discussion}

The Size of the images will be varied based on with color or without color. Wait bar is also used to take the entire images one by one basis. After taking collection of images we normalized all the images for getting unique input. Because, suppose we take group of white images, each and every image has a different background, different contrast, and different brightness. Because of this identification gets complicated. So we normalized all the images for getting same background, brightness, contrast etc. Wait bar is also used here for getting normalized images as one by one. Now we extract some of the features of the image using princomp function. The advantage of princomp function is it has taken some of the important features and extracts that features. We need not mention any particular feature. Then we test one particular test image with the collection of database images. Test images also normalized for getting unique images. Then only we easily compare the particular test image with database image, Otherwise comparison will be getting more complicated. After comparison the test image will be matched with the any one of the collection of database images, the person is authorized person.

The distance between the two images will be calculated using the above formula. If the distance between two images will be low means both the images have some same features between them. If the distance will be large between both images, it has some contrast features. So, both the images will not be coinciding. Then we set some threshold value. We easily identified the unauthorized person from the threshold value. After this testing process this mat lab coding will be interfaced with the system. The collection of input database is shown in figure 2 .

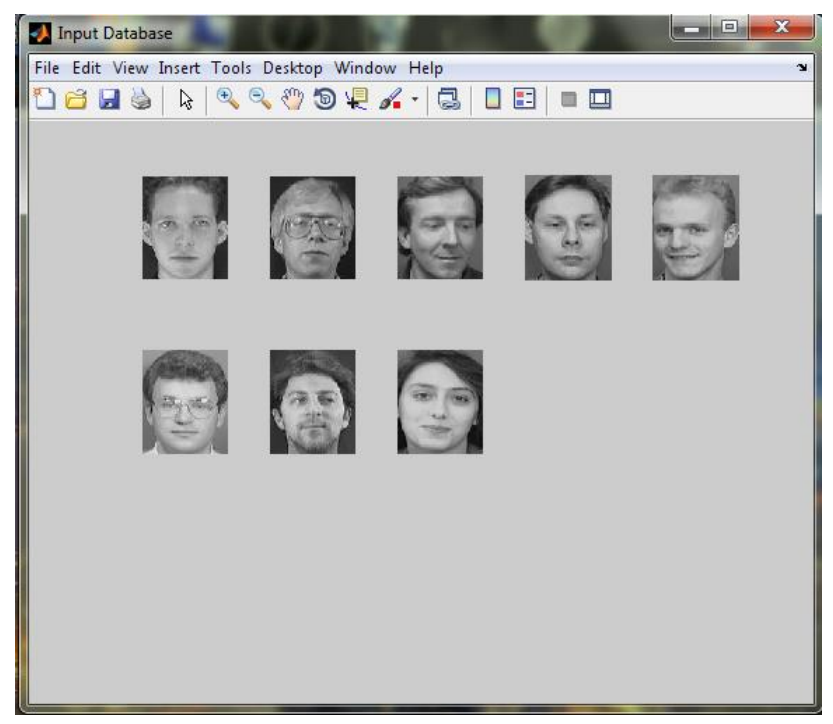

Fig.2: Collection of Input database 
The distance value is less than or equal means the images are matched. Suppose the distance value exceeds the threshold value means images are not matched. We easily identified unauthorized person. The distance is calculated using Euclidean formula. The normalized input is shown in figure 3.

$$
D=\operatorname{sqrt}((d 1+d 2) \wedge 2+(d 1-d 2) \wedge 2)
$$

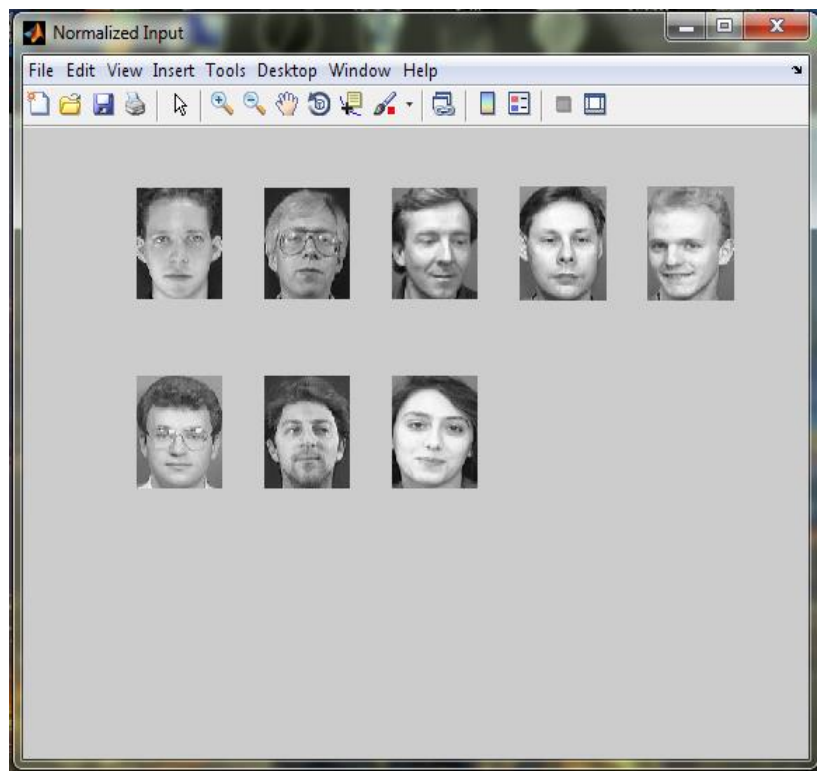

Fig. 3: Normalized image of the input

Suppose the test image is not matched with collection of database images the unauthorized person easily identified. This program coding will be done by using Matlab. Here the comparison will be done by the calculating the distances between the images and also a setting a threshold value for an images. Here the distance between the test image and matched database image is calculated. We set a threshold value for each and every image. The normalized test image and the matching results are shown in figure 4 and 5 respectively.

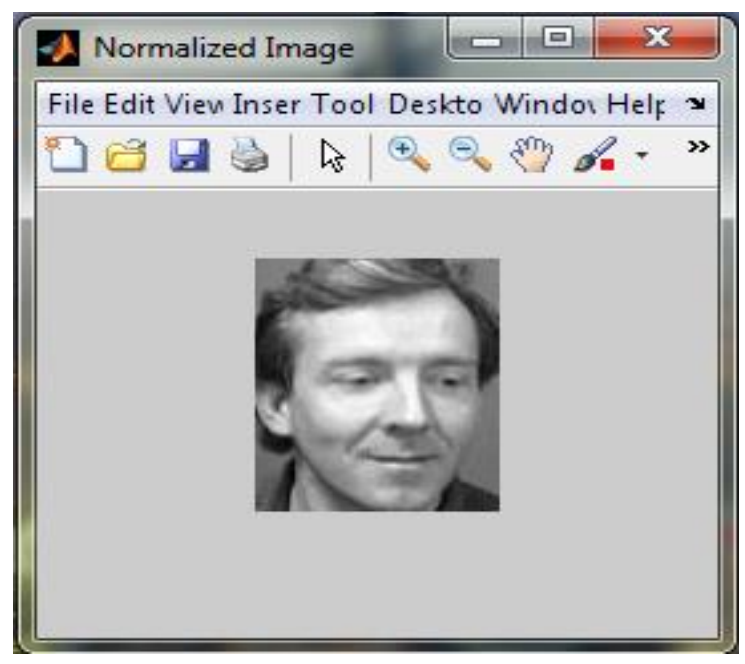

Fig.4: Normalized test Image 


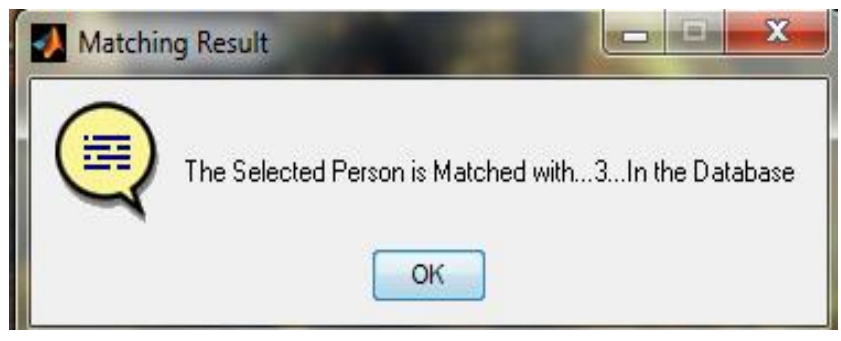

Fig.5: Matching Result

\section{Conclusion}

In digital image processing we identified unauthorized person with the use of Mat lab coding and princomp function. Through camera we find whether the detected obstacle is a human or not. If it is a human Mat lab coding compare both images, if it is matched we get the message through GSM modem. This is the effective human detection system. We easily identified unauthorized person using this system and also get notification message through GSM Modem. This is a low cost system; we get nearly accurate results through this system. This type of system provides more security and can provide applicability at banks, offices and secure places where the access to a system should be maintained more secure.

\section{References}

[1] Abu Sajana. R, Subramanian, Kumar P.V, Krishnan. S, "A low-complexity algorithm for intrusion detection in a PIR-based Wireless Sensor Network",IEEE Trans,vol.10,pp.6-21,2009.

[2] G. L. Graham, "Detection and classification for unattended ground Sensors,"IEEE Journal, vol.23, pp. 419424, Dec 2009.

[3] Hariprakash, R., Ananthi,S. Padmanabhan.K "An economical wireless network monitored scheme for camera based intrusion detection at unattended sites ”, IEEE Trans,vol.10,pp.29-36, Oct 2011.

[4] L. Peck and J. Lacombe, "Seismic-based personnel detection," in Proc. IEEE Sensors Journal, pp. 169175,2009 .

[5] Piero Zappi, Elisabetta Farella, and Luca Beniniand " Tracking Motion Direction and Distance With Pyro electric IR Sensors”, IEEE Sensors Journal,vol.10,pp.143-157, Sep 2010.

[6] Redmond,.S.J , K. D. Wong "Software simulation of unobtrusive falls detection at night-time using passive infrared and pressure mat Sensors",IEEE Journal, vol.8,pp.90-107,Mar 2010.

[7] Soumalya Sarkar, Asok Ray, Fellow "Target Detection and Classification Using Seismic and PIR Sensors" IEEE Sensor Journal, vol. 12,pp.66-78, June 2012.

[8] F. Xu and F. Xu, "Pedestrian Detection Based on Motion Compensation and HOG/SVM Classifier," 2013 5th International Conference on Intelligent Human-Machine Systems and Cybernetics, Hangzhou, 2013, pp. 334-337.

[9] E. D. Engeberg, M. Vatani and J. Choi, "Detection of the direction and speed of motion of forces on the surface of a compliant tactile sensor," 2013 13th International Conference on Control, Automation and Systems (ICCAS 2013), Gwangju, 2013, pp. 158-163.

[10] Q. Ma, "Motion detection system for HRI based on image parameters and HRI context," 2013 IEEE International Conference on Robotics and Biomimetics (ROBIO), Shenzhen, 2013, pp. 1288-1293.

[11] A. Yazar, A. E. Çetin and B. U. Töreyin, "Human activity classification using vibration and PIR sensors," 2012 20th Signal Processing and Communications Applications Conference (SIU), Mugla, 2012, pp. 1-4.

[12] X. Jin, S. Sarkar, A. Ray, S. Gupta and T. Damarla, "Target Detection and Classification Using Seismic and PIR Sensors," in IEEE Sensors Journal, vol. 12, no. 6, pp. 1709-1718, June 2012. 